\title{
Laser Induced Graphite Oxide/Graphene Transformation
}

\author{
Romualdas Trusovas $^{* 1}$, Gediminas Račiukaitis ${ }^{* 1}$, Jurgis Barkauskas ${ }^{{ }^{* 2}}$ and Regina Mažeikiene ${ }^{* 3}$ \\ ${ }^{*}$ Department of Laser Technologies, Center for Physical Sciences and Technology, Savanoriu Ave. \\ 231, Vilnius, LT-02300, Lithuania \\ romualdas.trusovas@ar.fi.lt \\ ${ }^{* 2}$ Faculty of Chemistry, Vilnius University, Naugarduko 24, LT-03225 Vilnius, Lithuania \\ ${ }^{*}$ Institute of Chemistry, Center for Physical Sciences and Technology, A. Gostauto 9, LT-01108 Vil- \\ nius, Lithuania
}

\begin{abstract}
In our experiments we aimed to structure the graphite oxide film by converting it locally into graphene by laser irradiation. An effect of ambient atmosphere on properties of the reduced graphene was also investigated. The reduction results were evaluated by measurements of electrical resistance and using Raman spectroscopy. The defect line at $1350 \mathrm{~cm}^{-1}$ remained still active in Raman spectra indicating disorder at edges of graphene sheets. A few-layer graphene can be locally formed in the film by direct laser writing, however stitching graphene flakes together remains as a problem that could be solved by incorporation of special organic molecules.

DOI:10.2961/jlmn.2012.01.0009
\end{abstract}

Keywords: Graphite oxide, reduction, graphene, laser irradiation, Raman spectra,

\section{Introduction}

Carbon is one of the most interesting elements of the periodic table. Carbon atoms are able to form many spatial configurations, some known for ages as graphite and diamond, and lately discovered fullerenes and nanotubes. Graphene is the most recently discovered allotrope of carbon, the two dimensional crystal. Due to its unique geometry graphene has distinguished physical features which can be used in various applications. This material has semiconductor properties, is mechanically strong and has a high thermal conductivity. It can be used in such applications as gas sensors, transistors, integral circuits, transparent electrodes.

Today there are many processes used to obtain graphene from different precursors such as chemical vapor deposition, electrostatic force assisted exfoliation [1], pulsed laser ablation of graphite on silicon [2] etc. There is a need in developing new reliable manufacturing processes for production of graphene on a macro scale. One of the ways is reduction of graphite oxide (GO) to graphene. Graphite oxide (GO) is widely used in the technology of preparation of graphene coating technology. This material has been well known since the mid-19th century, but its revival came now, especially with the increased interest in graphene. The name "graphite oxide" means a product of oxidation of graphite and but not name the individual chemical (similar to the polyethylene includes a complete set with a long chain saturated hydrocarbon molecules). Despite intensive research in recent years, the structure of this compound is relatively little known. There are many theories to explain the structure of GO [3]. Synthesis of GO results in an increase of the interlayer spacing between the oxygen-containing graphene layers.

Contrast in the thermal conductivities of GO and graphene can be beneficial for many practical applications, especially for the circuits with efficient heat transfer.
Moreover, laser irradiation can be used to convert the graphite oxide film locally into graphene by direct laser writing $[4,5,6]$, and even complex micro-scale graphene patterns can be produced from GO on a substrate [7]. The aim of this work is to use the laser treatment to form the thermo-conductive graphene-containing domains on the surface of thermal insulating GO coatings.

\section{Synthesis and film preparation of graphene oxide}

Samples of graphite oxide (GO) were synthesized in the Laboratory of Carbonaceous Materials, Vilnius University using the standard Hummers - Otieman [8] and modified synthesis protocols. GO coatings of different thickness (20$1200 \mathrm{~nm}$ ) were produced on polymer membranes using novel preparation methods developed in the laboratory. Some coatings were treated with the organic reagents containing certain functional groups, which were supposed to join up isolated GO sheets.

During this work, graphite oxide synthesis technique was developed and modified versions of this synthesis were created, intermediate and final products of GO synthesis were analyzed. Synthesized products were modified using functional reagents. Using the GO and its modified products, a graphite oxide coatings polymer processing technique was developed, and analysis of substrates for these coatings was performed.

Two types of membrane filters with different a pore size and texture were selected for the experiments: Polycarbonate (PC) and Nylon (MagnaNylon) with the mean pore diameter of $0.22 \mu \mathrm{m}$ and $0.45 \mu \mathrm{m}$. After making a series of tests, it was found that the highest quality of the GO coatings was achieved on the polycarbonate membrane filters. The membrane filters with a rough surface can be used only as a substrate in formation of thick GO layers of sufficient quality. 
An important step was the preparation of water-based coating suspensions for GO production. GO disperses the aqueous phase relatively easy, without need of using additional chemical dispersing agents. For fabrication of coatings $5^{*} 10^{-5} \mathrm{~g} / \mathrm{ml}$ suspensions, which were prepared from a higher concentration $\left(5^{*} 10^{-3} \mathrm{~g} / \mathrm{ml}\right)$ with dilution, were used. Before the suspensions were filtered they were sonificated for 30 minutes (Sonic VibraCell, $12 \mathrm{~mm}$ diameter electrode, $13 \%$ amplitude). GO state in solution is sensitive to a base of suspension (Fig. 1). Neutral and basic GO suspension (with steady-state concentration of KOH $0.02 \mathrm{M}, 0.002 \mathrm{M}$ and $0.0002 \mathrm{M}$ ) was used for coating preparation.

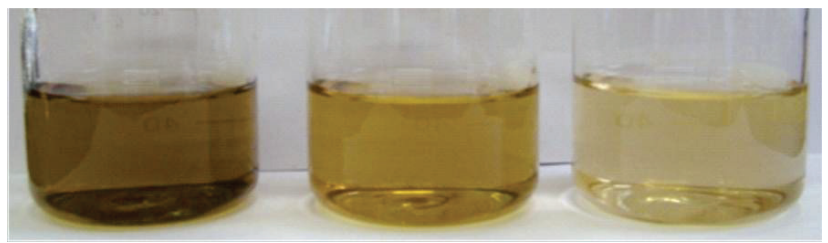

Fig. 1. GO suspension with different steady-state concentration of $\mathrm{KOH}$ (from left to right): $0.02 \mathrm{M}, 0.002 \mathrm{M}, 0.0002 \mathrm{M}$.

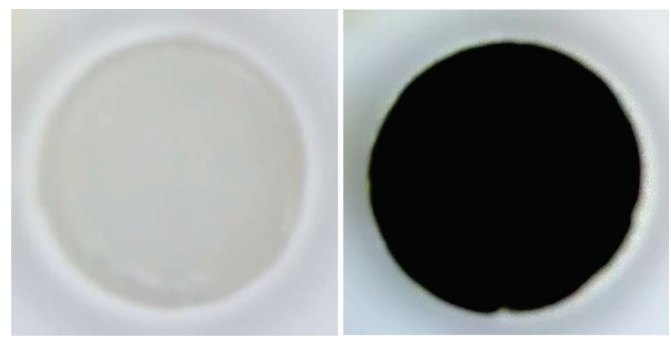

Fig. 1. GO coatings prepared on a polycarbonate substrate. Thickness of the GO layer: $40 \mathrm{~nm}$ (left) and $400 \mathrm{~nm}$ (right).

Coatings of GO and its modified products were formed by filtration of aqueous suspensions through the membrane filters in an aqueous medium. The $\mathrm{pH}$ of aqueous media was chosen as acidic, neutral or alkaline. In such manner it was intended to disrupt the stability of aqueous suspensions to form coatings of $\mathrm{GO}$ and its modified products, on the membrane filters. Using of polycarbonate filters enables preparation of relatively thin GO coating (up to $20 \mathrm{~nm}$ ) (Fig. 2). Coatings of modified GO products were also prepared using this method.

\section{Laser treatment}

Experiments on reduction of graphite oxide to graphene were performed using localized laser heating. The picosecond laser (PL10100, 10 ps, $100 \mathrm{kHz}$, Ekspla) and the nanosecond laser (NL15100, $10 \mathrm{ns,} 20 \mathrm{kHz}$, Ekspla) were used in experiments. Experimental setup with a picosecond laser included the laser, electro-optical shutter, nonlinear crystal for wavelength conversion, the beam expander and galvanometer scanners with focusing objectives for both $1064 \mathrm{~nm}$ and $532 \mathrm{~nm}$ wavelengths (Fig. 3). Experimental setup with a nanosecond laser included the laser, nonlinear crystal for wavelength conversion, the beam expander and the galvanometer scanner for the $532 \mathrm{~nm}$ wavelength.

The line patterns were formed in GO films by scanning a focused laser beam. During the tests, the average laser power was varied from $1 \mathrm{~mW}$ to $100 \mathrm{~mW}$. Scanning speeds were changed from $1 \mathrm{~mm} / \mathrm{s}$ to $1000 \mathrm{~mm} / \mathrm{s}$ to see the effect of single and high overlap of laser pulses (99\%). Experiments were conducted in air, nitrogen or argon atmosphere.

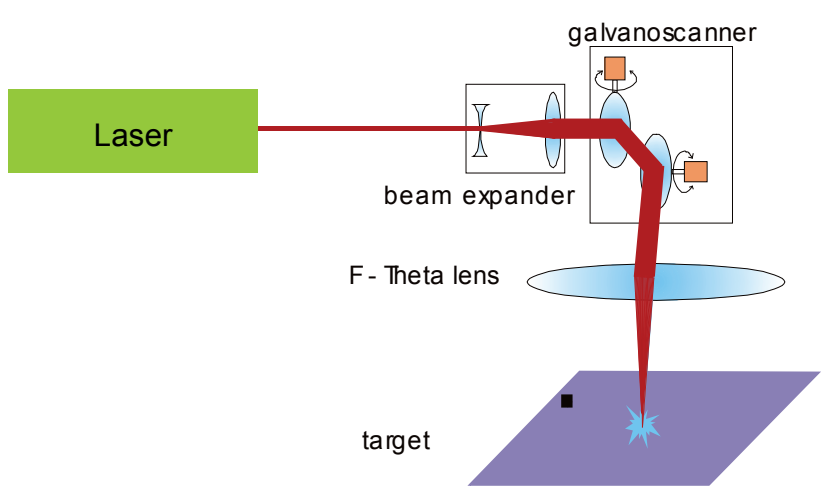

Fig. 2. Experimental setup for laser treatment of graphite oxide.

Irradiation with the laser beam caused a change in morphology of the GO film. After laser processing, samples were at first investigated under the optical microscope (Olympus BX51TF). Further evaluation of the laser processed samples included scanning electron microscopy, measurement of electrical resistance and Raman spectroscopy.

Even that we conducted the experiment with infrared $(1064 \mathrm{~nm})$ and green $(532 \mathrm{~nm})$ laser radiation, in case of both used lasers (nanosecond and picosecond) the green radiation caused burning of the GO layer independently of process parameters. Therefore, the results presented below were achieved using the picosecond laser working at a fundamental wavelength.

Using $100 \mathrm{~mW}$ and higher average laser power, depending on the thickness of the sample and the scanning speed of the graphite oxide layer, the scanned areas burned out using the infrared laser as well. Using the average laser power below $10 \mathrm{~mW}$ did not show visible effects of the laser even in the thinnest samples, which were $10-40 \mathrm{~nm}$ in thickness.

A scanning electron microscope (SEM) (JSM-6490 LV, JEOL) was used for more precise evaluation of surface modifications of laser treated GO films. Some SEM images are presented below.

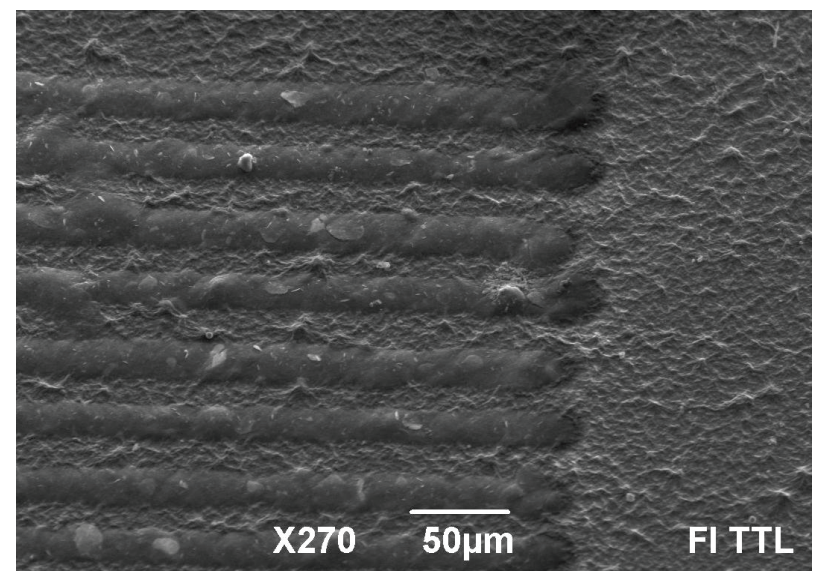

Fig. 3. SEM image of laser modified lines in GO film. Process parameters: laser PL10100, wavelength $1064 \mathrm{~nm}$; irradiation dose $6.37 \mathrm{~J} / \mathrm{cm}^{2}$ (laser power $10 \mathrm{~mW}$; scanning speed $10 \mathrm{~mm} / \mathrm{s}$ ); ambient air. Sample: aqueous GO suspension, concentration. $1.5 * 10^{-4}$ $\mathrm{g} / \mathrm{ml}$; filtrated to $0.1 \mathrm{M} \mathrm{KOH}, \mathrm{PC}$ substrate (pore $\mathrm{d}=0.4 \mu \mathrm{m}$ ); 
doped by Congo red dye $0.001 \mathrm{M}, 10 \mathrm{~mL}$; Film thickness $1200 \mathrm{~nm}$.

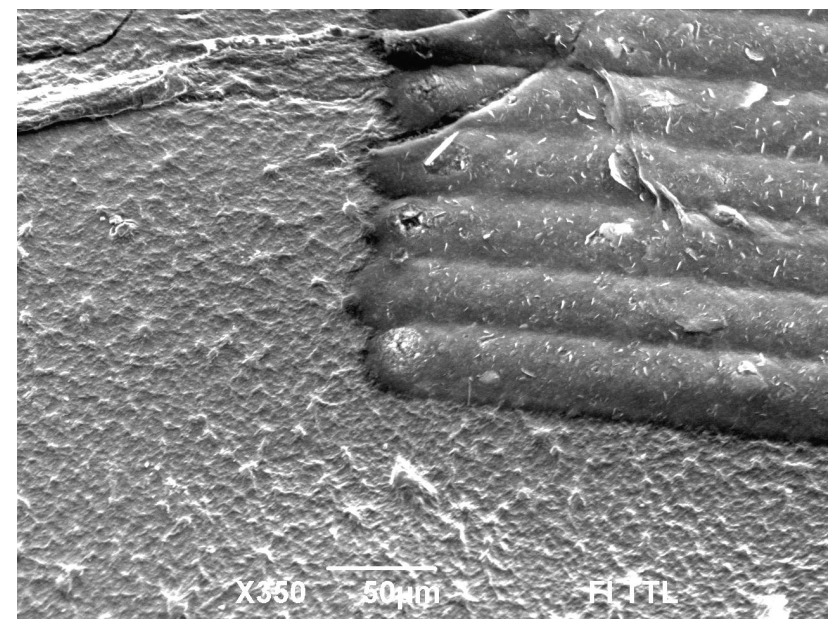

Fig. 4. SEM image of laser modified lines in GO film. Process parameters: laser PL10100, wavelength $1064 \mathrm{~nm}$; irradiation dose $6.37 \mathrm{~J} / \mathrm{cm}^{2}$ (laser power $30 \mathrm{~mW}$; scanning speed $30 \mathrm{~mm} / \mathrm{s}$ ); ambient air. Sample: the same as for Fig. 4.

Fig. 4 and Fig. 5 present laser modified lines in GO film doped with Congo red dye. The laser processing parameters are quite similar (the same irradiation dose: the laser power divided by the scanning speed). Surface of GO film was smoothened when the low laser power at low scanning speed were used. However, in case of higher laser power even at a higher scanning speed, smoothening of the surface was accompanied by cylindrical exfoliation of the graphite oxide layer. Keeping the same laser processing parameters but in nitrogen atmosphere, a visual effect of laser processing was similar (Fig. 6). A further increase in the mean laser power at the same scanning speed led to damage of the GO film in the center of laser scan line where intensity of the laser beam was maximal due to the Gaussian distribution of the beam.

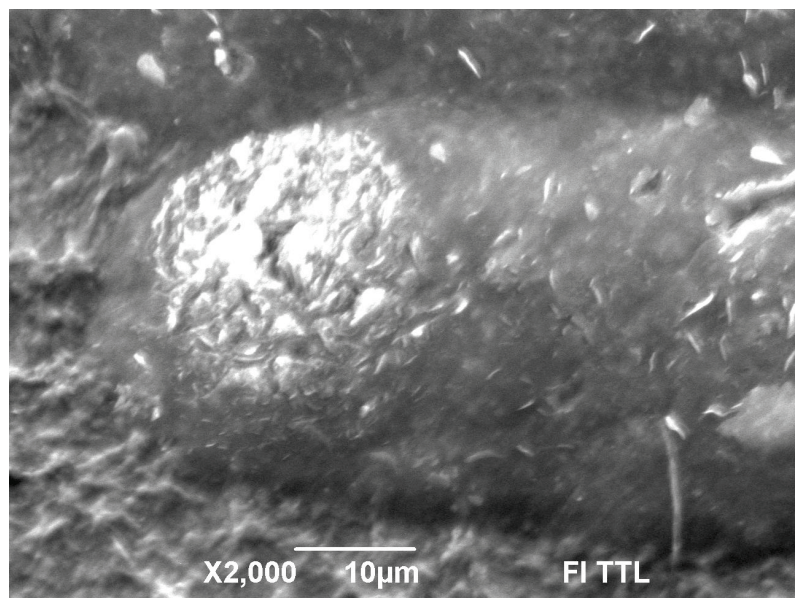

Fig. 5. SEM image of laser modified lines in GO film. Process parameters: laser PL10100, wavelength $1064 \mathrm{~nm}$; irradiation dose $6.37 \mathrm{~J} / \mathrm{cm}^{2}$ (laser power $30 \mathrm{~mW}$; scanning speed $30 \mathrm{~mm} / \mathrm{s}$ ); nitrogen atmosphere. Sample: the same as for Fig. 4.

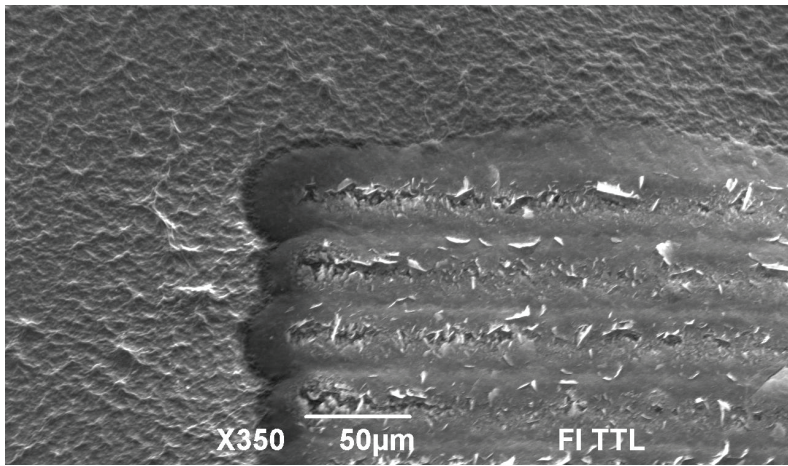

Fig. 6. SEM image of laser modified lines in GO film. Process parameters: laser PL10100, wavelength $1064 \mathrm{~nm}$; irradiation dose $10.62 \mathrm{~J} / \mathrm{cm}^{2}$ (laser power $50 \mathrm{~mW}$; scanning speed $30 \mathrm{~mm} / \mathrm{s}$ ); nitrogen atmosphere. Sample: aqueous GO suspension, concentration. $1.5^{*} 10^{-4} \mathrm{~g} / \mathrm{ml}$; filtrated to $\mathrm{H}_{2} \mathrm{O}, \mathrm{PC}$ substrate (pore $\mathrm{d}=0.4 \mu \mathrm{m}$ ); doped with Alkali blue dye $0.001 \mathrm{M}, 0.5 \mathrm{~mL}$; Film thickness $1200 \mathrm{~nm}$.

The used dopants modified the GO film and laser processing results were different in the same range of parameters. Fig. 7 and Fig. 8 present SEM images of laser modified lines in the GO film doped with Alkali Blue dye.

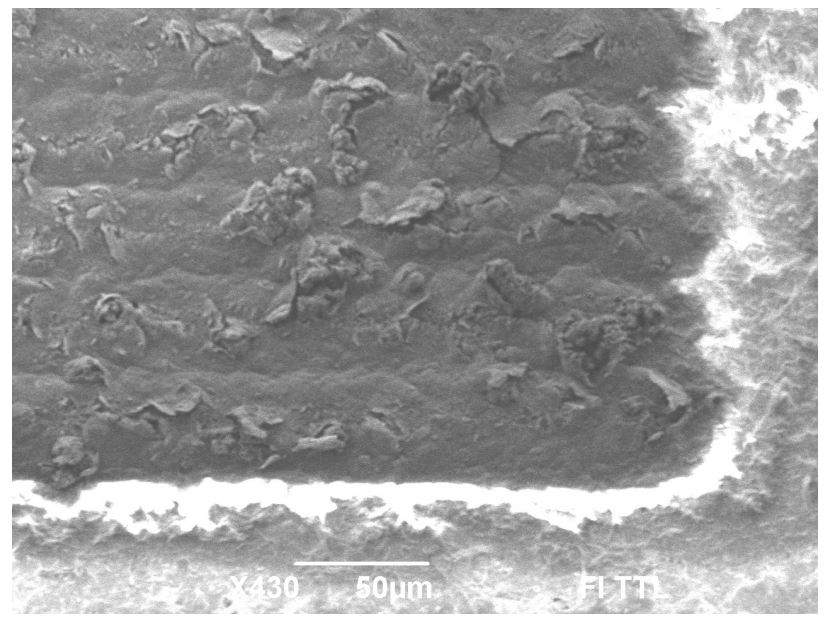

Fig. 8. SEM image of laser modified lines in GO film. Process parameters: laser PL10100, wavelength $1064 \mathrm{~nm}$; Irradiation dose $2.55 \mathrm{~J} / \mathrm{cm}^{2}$ (laser power $20 \mathrm{~mW}$; scanning speed $50 \mathrm{~mm} / \mathrm{s}$ ); argon atmosphere. Sample: the same as for Fig. 7.

The surface of laser modified areas in samples doped with the Congo red dye is smooth, while the surface of laser irradiated areas in Alkali Blue dye doped samples was notably damaged with irregular cracking patterns. The reason could in ability of Congo red to form larger moieties composed of the nanoplatelets of graphene due to interaction between the amino groups in Congo red molecule and the functional groups of GO located on the edges of nanoplatelets.

\section{Electrical resistance of modified GO layer}

Graphite oxide and graphene possess completely different electrical and related thermal conductivity properties. $\mathrm{GO}$ is an isolating material, while graphene is well known as a highly conductive one. Therefore, measurements of electrical resistance were used as one of the means to evaluate reduction of GO to graphene phase. The resistance was measured with an ohmmeter between edges of an array 
of laser beam modified lines of $1 \mathrm{~cm}$ in length. Results for the GO films with the initial thickness of 800-1200 nm are shown in Fig. 9.

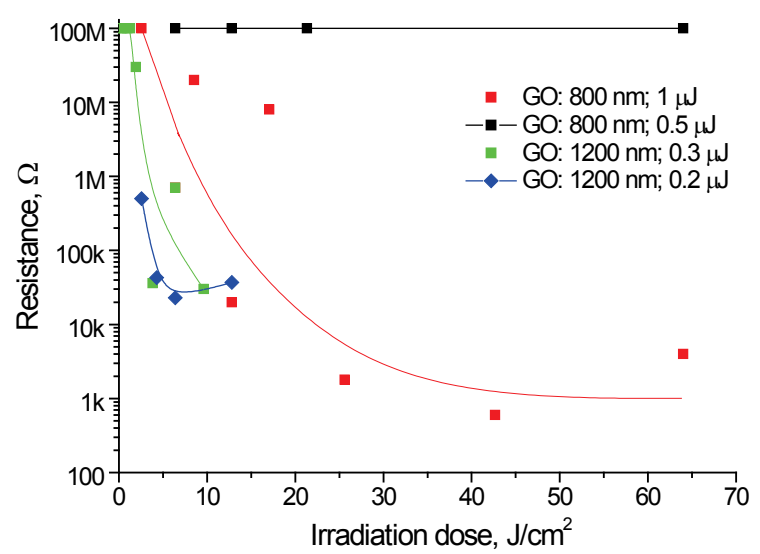

Fig. 9. Dependence of the GO film resistance on the irradiation dose for different GO films. Samples irradiated with the $1064 \mathrm{~nm}$ wavelength from the picosecond laser in air atmosphere. Solid lines are approximations of experimental data.

Resistance of the GO films irradiated with the picosecond $1064 \mathrm{~nm}$ laser decreased by 4-5 orders from 100s of megaohms to one kiloohm in a certain range of laser processing parameters. The irradiation dose was estimated from laser fluence in the center of the focused laser beam and beam overlap during scanning the beam across the surface. Rapid changes in the resistance were observed on the low dose side, while minimum in resistivity was also pronounced. For the GO film with thickness of $800 \mathrm{~nm}$ it was identified that the low pulse energy $(0.5 \mu \mathrm{J})$ did not cause changes in resistivity of the film independent of the accumulated dose.

\section{Raman spectra analysis}

Laser irradiated samples of GO films were analyzed using Raman scattering spectroscopy. This technique is considered to be one of the most reliable means in identifying graphene allotrope [7].

Raman measurements were performed with $632.8 \mathrm{~nm}$ excitation (He-Ne laser) by using the confocal Raman spectrometer/microscope LabRam HR800 (Horiba Jobin Yvon) equipped with a grating containing 600 grooves $/ \mathrm{mm}$ and a liquid nitrogen cooled $\mathrm{CCD}$ camera down to $-132^{\circ} \mathrm{C}$ working temperature. The laser power at the sample was restricted to $1 \mathrm{~mW}$ and the laser beam was focused to $\sim 2 \mu \mathrm{m}$ diameter spot on the surface. Spectra were taken with 50x objective lens. Raman measurements were carried out in $180^{\circ}$ geometry. The integration time was $10 \mathrm{~s}$. Each spectrum was recorded by accumulation of 10 scans yielding overall integration time of $100 \mathrm{~s}$. Raman spectra were captured from different areas of the samples: pristine GO films, which were not irradiated with the laser and from the center of laser scribed lines.

Most significant features of the spectra of graphitic materials were as follows (identification according to $[9,10])$ :

- G-band $1582 \mathrm{~cm}^{-1}$

- D-band $1350 \mathrm{~cm}^{-1}$
- D'-band in $1620 \mathrm{~cm}^{-1}$

- 2D-band $2700 \mathrm{~cm}^{-1}$

The G-band corresponds to the $\mathrm{sp}^{2}$-bonds of carbon atoms in the material (in-plane $E_{2 g}$ vibrational mode) [11]. This type of bonds forms graphite and graphene. The $\mathrm{D}$ and D'-bands correspond to the $\mathrm{A}_{1 \mathrm{~g}}$ breathing mode and are considered to be related to defective arrangement of carbon atoms. For this reason, the D-band should disappear in Raman spectra of highly oriented graphene layers without defects [12]. The intensity ratio of the Raman bands $\mathrm{I}_{\mathrm{D}} / \mathrm{I}_{\mathrm{G}}$ is widely used to quantify defects in graphitic materials. The 2D-band is the overtone of the D-band. Its shape indicates the number of layers of graphene, and therefore the line can also be used for the $\mathrm{I}_{2 \mathrm{D}} / \mathrm{I}_{\mathrm{G}}$ ratio [9].

Examples of the Raman spectra captures on the sample processed at various laser and scanning parameters are shown in Fig. 10.

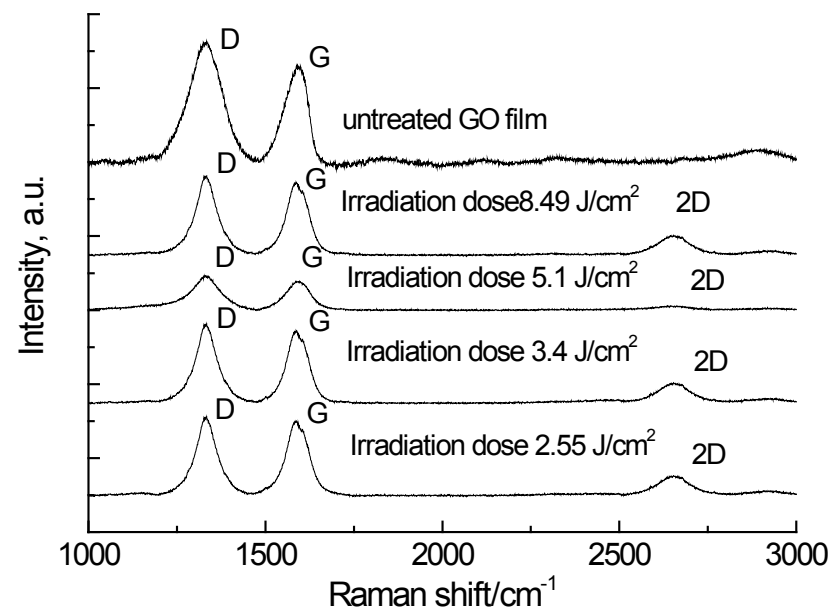

Fig. 10. Raman spectra of unaffected GO film and various scanning parameters using $1064 \mathrm{~nm}$ radiation in nitrogen atmosphere

Sample: $0.02 \mathrm{M} \mathrm{KOH}+\mathrm{GO}$ suspension, concentration. $5 * 10^{-5}$ $\mathrm{g} / \mathrm{ml}$; Sonification for $30 \mathrm{~min}$; Coating thickness $400 \mathrm{~nm}$, Filtrated to $0.1 \mathrm{M} \mathrm{KOH}, \mathrm{PC}$ substrate (pore $\mathrm{d}=0.45 \mu \mathrm{m}$ ).

Depending on the laser treatment parameters, the ratio $\mathrm{I}_{\mathrm{D}} / \mathrm{I}_{\mathrm{G}}$ decreased 10 times, and the 2D-band, indicating graphene layers formation, appeared [13]. However, the D-line was observed in Raman spectra of all laser irradiated GO films, used in the investigations. This spectral line shows presence of packing irregularities [9] and the reason can be presence of oxygen during the experiments. The D-line was slightly reduced by irradiating GO in argon atmosphere [14]. G line shift to the higher wave numbers can be recognized in the spectra with higher 2D-band intensities. Such shift corresponds to the decrease in the number graphene layers. [15]. Therefore, we also conducted experiments in an evacuated chamber filled with nitrogen or argon gas. For samples, irradiated with the picosecond laser in nitrogen or argon atmosphere, the $\mathrm{I}_{\mathrm{D}} / \mathrm{I}_{\mathrm{G}}$ ratio was lower than in air atmosphere at the same laser treatment parameters. For more demonstrative identification of most suitable laser treatment parameters $\mathrm{I}_{2 \mathrm{D}} / \mathrm{I}_{\mathrm{G}}$ ratios were calculated (Fig. 11). The highest value of this ratio means the best reduction results. According to this evaluation better results are achieved in argon and nitrogen atmosphere, when the irradiation dose was $6.37 \mathrm{~J} / \mathrm{cm}^{2}$. 


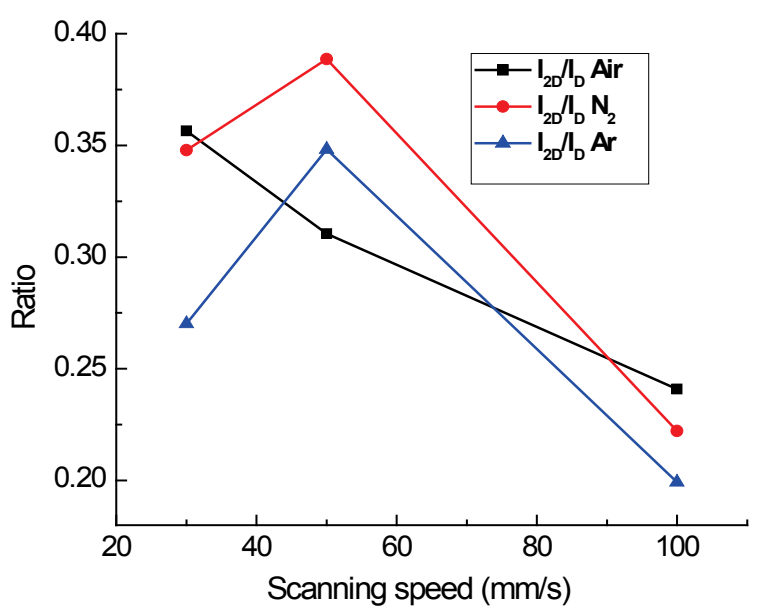

Fig. 11. The ratio of intensities in Raman lines $I_{2 D} / I_{D}$ versus scanning speed at the constant mean laser power of $50 \mathrm{~mW}$ $\left(\mathrm{F}=0.16 \mathrm{~J} / \mathrm{cm}^{2}\right)$ in air, nitrogen and argon atmosphere. Experiments were conducted with $1064 \mathrm{~nm}$ radiation. Sample: $\mathrm{H}_{2} \mathrm{O}$ GO suspension, concentration. $5^{*} 10^{-4} \mathrm{~g} / \mathrm{ml}$; suspension with Alkali blue $0.001 \mathrm{M}, 0.5 \mathrm{ml}\left(\mathrm{c} 5^{*} 10^{-6} \mathrm{M}\right)$; sonificated $1 \mathrm{~h}$; Coating thickness $1200 \mathrm{~nm}$, Filtrated to $\mathrm{H}_{2} \mathrm{O}$, PC substrate (pore $\mathrm{d}=0.4 \mu \mathrm{m}$ ).

Similar behavior was observed for the GO film doped with Congo red. The highest ratio between intensities of Raman lines $\mathrm{I}_{2 \mathrm{D}} / \mathrm{I}_{\mathrm{D}}$ was achieved when laser irradiation was performed in oxygen-free atmosphere (Fig. 12), however the optimal laser processing parameters shifted to the lower mean laser power, keeping the same irradiation dose.

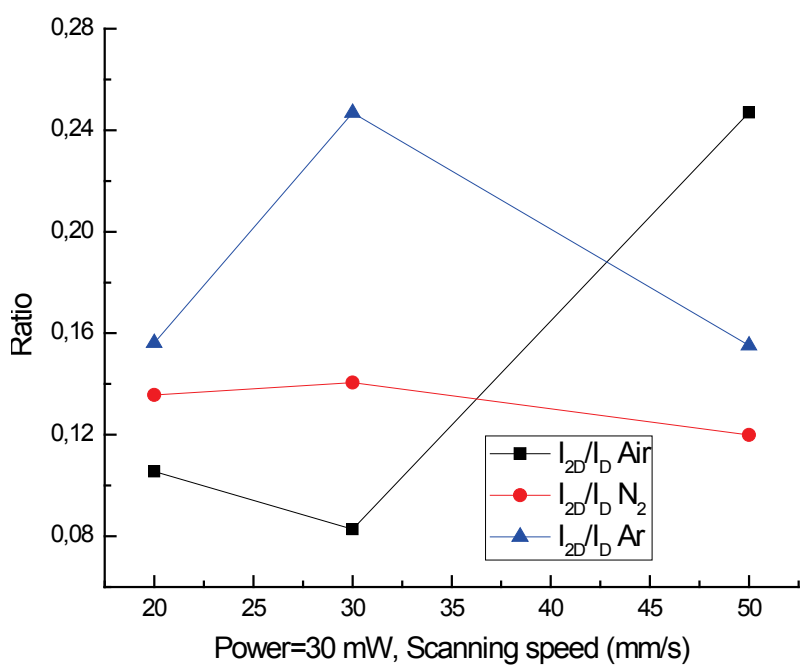

Fig. 12. The ratio of intensities in Raman lines $I_{2 D} / I_{D}$ versus scanning speed at the constant $30 \mathrm{~mW}$, power $\left(\mathrm{F}=0.1 \mathrm{~J} / \mathrm{cm}^{2}\right)$ in air, nitrogen and argon atmosphere.. Experiments were conducted with $1064 \mathrm{~nm}$ radiation. $\mathrm{H}_{2} \mathrm{O}$ GO suspension, concentration. $5 * 10^{-4} \mathrm{~g} / \mathrm{ml}$; Suspension with Congo red $0.001 \mathrm{M}, 5 \mathrm{ml}\left(\mathrm{c} 5 * 10^{-5}\right.$ M) Sonificated $1 \mathrm{~h}$; Coating thickness $1200 \mathrm{~nm}$, Filtrated to $\mathrm{H}_{2} \mathrm{O}$, PC substrate (pore $\mathrm{d}=0.4 \mu \mathrm{m}$ );

\section{Conclusions}

Summarizing the experiments on laser reduction of graphite oxide to graphene, the picosecond laser generating at $1064 \mathrm{~nm}$ wavelength initiated conversion of the material. The processing regimes were evaluated based on measurement of electrical resistance and Raman scattering spectra. The optimal mean laser power was in the range of 10-
$100 \mathrm{~mW}$ (laser fluence in the range of $\mathrm{F}=0.01-0.3 \mathrm{~J} / \mathrm{cm}^{2}$ ), depending on the sample thickness and preparation. The optimum scanning speed was from 10 to $50 \mathrm{~mm} / \mathrm{s}$ at the $100 \mathrm{kHz}$ repetition rate.

Resistance of the GO films irradiated with the picosecond $1064 \mathrm{~nm}$ laser decreased by 4-5 orders in a certain range of laser processing parameters and the 2D-line appeared in Raman spectra of same samples after laser treatment in ambient air indicating formation of graphene phase By conducting additional experiments in nitrogen and argon atmosphere we succeeded to achieve the higher $\mathrm{I}_{2 \mathrm{D}} / \mathrm{I}_{\mathrm{D}}$ ratio in Raman spectra of laser modified samples.

However, the D-line still remained in Raman spectra and further development of GO coatings is required for avoiding structural defects in graphene phase after local reduction by laser irradiation.

\section{Acknowledgments}

This research was funded by a grant No. ATE-06/2010 from the Research Council of Lithuania.

\section{References}

[1] Y. Zhou, Q. Bao: Adv. Mater. 21, (2009) 1.

[2] E. Cappelli, S. Orlando: J. Phys.: Conf. Series, 59, (2007) 616.

[3] D. R. Dreyer, S. Park, C. W. Bielawski, R. S. Ruoff: Chem. Soc. Rev. 39, (2010) 228.

[4] 1. Z.B. Liu. ${ }^{1}$, L. Li. ${ }^{1}$, Y.F. Xu, J.J. Liang, X. Zhao, S.Q. Chen, Y.S. Chen, J.G. Tian: J. Optics 13 (2011) 085601 .

[5] D.E. Sokolov, K.R. Shepperd, T.M. Orlando: J. Phys. Chem. Lett. 1, (2010) 2633.

[6] Y. Zhang, L. Guo, S. Wei, Y. He, H. Xia, Q. Chen, H. Sun: Nano Today 5, (2010) 15-20.

[7] D. Graf, F. Molitor: Eur. Phys. J. Special Topics 148, (2007) 171.

[8] G. Brauer. Handbuch der präparativen anorganischen Chemie. B3, Ferdinand Enke Verlag, Stuttgart, 1975.

[9] Sh. Amini, J. Garay: J. Appl. Phys. 108, (2010) 093105 .

[10] C. Casiraghi, S. Pisana, K.S. Novoselov, A. K. Geim, A. C. Ferrari: Appl. Phys. Lett. 91, (2007) 233108.

[11] D. Zhan, Z. Ni, W. Chen, L. Sun, Z. Luo, L. Lai, T. $\mathrm{Yu}, \mathrm{A}$. Thye, S. Wee, Z. Shen: Carbon 49, (2011) 1362.

[12] C. Faugeras, A. Nerriere, M. Potemski, A. Mahmood, E. Dujardin, C. Berger, W. A. de Heer: Appl. Phys. Lett. 92, (2008) 011914

[13] A. Gupta, G. Chen, P. Joshi, S. Tadigadapa, P.C. Eklund: Nano Letters, 6, (2006) 2667.

[14] J. Rohl, M. Hunhausen, M. Emstev, Th. Seyller, R. Graupner, L. Ley: Appl. Phys. Lett. 92, (2008) 201918.

[15] D. Graf, F. Molitor, K. Ensslia, C. Stampfer, A. Jungen, C. Hierold, L. Wirtz: Solid State Comm. 143, (2007) 44.

(Received: June 7, 2011, Accepted: December 26, 2011) 